\title{
Mythos, Mühsal, Machbarkeit
}

Klaus-D. Liedke

\begin{abstract}
Um soziale Dienste wirklich steuern und lenken zu können, bräuchte es eine Theorie sozialer Dienstleistung. Diese fehlt zwar noch, aber Elemente dazu gibt es bereits.
\end{abstract}

Sozialarbeit kennt viele Fachgebiete und Betriebsformen und keiner besitzt ein Universalrezept, wie das alles so richtig funktioniert. Eine allgemeingültige Theorie sozialer Dienstleistung gibt es bislang nämlich nicht. Doch es wäre schon gut zu wissen, wie sich das sensible Gut effektiv bewirtschaften lässt, wie man humane Dienste verdeutlichen und fair bemessen kann, sie nach dem Bedürfnis der Menschen und dem Bedarf der Gemeinschaft ausrichten, nach betrieblichem Vermögen und seinen Zielen lenken usw. triebliche Vorgänge zu strukturieren und zu verwalten, Tätigkeiten der Mitarbeiter und Kunden zu dokumentieren, nötige Folgehandlungen $\mathrm{zu}$ veranlassen und $\mathrm{zu}$ lenken und vieles andere mehr.

\section{Warum bloß ist Soziale Arbeit so schwer zu fassen?}

Soziale Aktivitäten sind in der Praxis nicht einfach zu organisieren. Denn das mit der Hilfeleistung verbundene Geschehen ist wenig systematisch angelegt und man muss sich oft mit partiellen Lösungen behelfen. Zwar sind auch andere Güter nicht immer trivial, aber sozialwirtschaftliche Tätigkeiten sind doch mit besonderen Schwierigkeiten verbunden, schwer zu verstehen. Denn wie funk-

\section{"Was zählt eigentlich bei sozialen Dienstleistungen: was man aufwendet, was geschieht, was herauskommt? "}

Mit Fragen der Steuerung sozialer Hilfen befassen sich viele Fachverbände, Leistungsträger und Sozialplaner. Vor allem aber müssen sich Sozialbetriebe selbst damit auseinandersetzen, denn schließlich erbringen sie die Leistungen und müssen allerlei Aufgaben erfüllen, die sich dabei stellen. Auch LEBENSRÄUME in Offenbach, ein auf psychosoziale Hilfen spezialisierter Träger, arbeitet daran, sein breites Leistungsportfolio gut $\mathrm{zu}$ managen. Es geht darum, Ziele und Mittel der diversen Hilfeleistungen zu balancieren, dazu erforderliche be- tionieren eigentlich beratende, begleitende oder therapeutische Dienste und was zählt dabei: Was man aufwendet, was geschieht, was herauskommt? Und worin unterscheiden sich Hilfeformen, wie hängen materielle, immaterielle und ideelle Bestandteile zusammen? Werden wirklich alle eingesetzten Dienste gebraucht und was ist mit dem, was übrig bleibt oder fehlt, mit Alternativen? Viele Fragen stellen sich und einige grundsätzliche Eigenschaften sozialer Hilfeleistung sollte man sich klarmachen, bevor es an Lösungen geht:

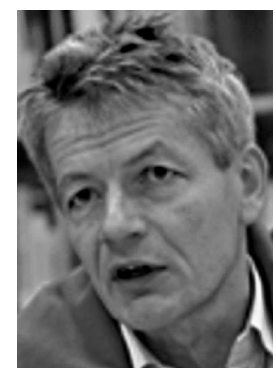

Klaus-D. Liedke ist 55 Jahre alt und lebt in Offenbach. Nach Lehre und Konstrukteurstätigkeit studiert er Sozialpädagogik in Frankfurt am Main und arbeitet seit 1982 im gerade gegründeten Psychiatrischen Hilfsverein LEBENSRÄUME in Offenbach in verschiedener Funktion. Seit 1991 führt er die Geschäfte des Vereins mit seinen Betriebsgesellschaften. Nach Abschluss eines Zweitstudiums der Betriebswirtschaftslehre 1998 in Lahr spezialisierte er sich auf Themen der sozialen Dienstleistung. Seit 2000 unterrichtet er an der Fachhochschule Wiesbaden im Aufbaustudiengang Diplom-Sozialmanagement im Sozial- und Gesundheitswesen, dessen Curriculum er zusammen mit Prof. Dr. Reinhard Peukert entwickelt hat.

E-Mail

Klaus-D.Liedke@leb-of.de

- Soziale Dienste sind ein persönliches Gut. Hilfeleistungen berühren die Menschen nicht nur unmittelbar, sondern sind eingebunden in Lebenssituationen mit familiären, gemeinschaftlichen, arbeitsbezogenen und anderen Bindungen, sind geprägt durch subjektive Vorstellungen der Betroffenen und Beteiligten. Soziale und psychosoziale Hilfe bestehen aus Motiven und Materiellem, aus Handlung und Haltung und das alles ändert sich ständig. Es fällt schwer, Regelmäßigkeit zu erkennen, planvoll vorzugehen, Standards zu setzen und manchmal ist das sogar unnütz oder schädlich.

- Soziale Dienste sind ein gemeinschaftliches Gut. Im sozialen und marktwirtschaftlichen Rechtsstaat sind Bürger für sich selbst verantwortlich, über die Solidargemeinschaft aber auch versichert gegen allerlei Lebensrisiken. Kulturelle Gewohnheiten und fachliche Erkenntnisse, politischer Wille und 
ökonomische Möglichkeiten der Gemeinschaft prägen soziale Hilfe und Wohlfahrtspflege hat dies alles nach rechtlichen Regeln, irgendwo zwischen staatlicher Fürsorge und reguliertem Wettbewerb umzusetzen. Solche Rahmenbedingungen prägen soziale Dienstleistung mehr als alles andere.

\section{- Soziale Dienste sind ein wirt-} schaftliches Gut. Auch in öffentlicher Trägerschaft sind soziale Hilfen ein ökonomischer Faktor, erfordern Haushaltsbudgets, Ämter usw. In der entwickelten Dienstleistungsgesellschaft fallen gemeinschaftliche Leistungen aber immer mehr an private Trägerorganisationen und wenn Sozialbetriebe diese Aufgaben übernehmen, müssen sie je nach Größe und Anspruch ein spezielles betriebswirtschaftliches Instrumentarium entwickeln: Industrialisierung sozialer Dienstleistung, hybride Serviceprodukte, Controlling im Leistungsmanagement sind moderne Schlagworte.

Man ahnt die Schwierigkeiten und es ist fast selbstverständlich, dass sich in diesem Komplex nicht alles rational und logisch steuern lässt. Die Kunst der Führung Sozialer Arbeit besteht vielleicht gerade darin, im immer irgendwie diffusen Gemenge das Machbare zu erkennen und zu verfolgen. Man kann sich Ziele setzen, aber braucht auch Möglichkeiten, sie umzusetzen. Und umgekehrt, Gelegen- heit schafft Absichten. Man darf Dinge nicht einfach gehen lassen und muss doch akzeptieren, wenn etwas anders wird. Das ist Soziale Arbeit.

Aber trotz der immensen Komplexität und Variabilität ist nicht alles nur zufällig und beliebig im professionellen Hilfegeschehen. Wenn man soziale Dienste absichtsvoll lenken will, mag es helfen, ihren Wesenselementen erst einmal etwas nachzuspüren.

\section{Das komplexe System lässt sich im Modell verstehen}

Als Folge vieler diffuser Eigenschaften der sozialen Dienste kommt man schnell vom einen zum anderen. Stehen fachliche Aspekte im Vordergrund, sind zugleich Ressourcen, Geld und andere Faktoren zu bedenken und wenn ganze Betriebe und Unternehmen betrachtet werden, ist doch schnell wieder das fachliche Geschick entscheidend.

Eine Hilfeleistung mag persönlich nötig, soll individuell beschaffen sein, aber die Maßstäbe dafür richten sich nach gesellschaftlicher Verträglichkeit, sozialrechtlichen Regeln und verfügbaren Mitteln. Erwartung und Intensionen der Beteiligten bewirken manchmal mehr, als alle dienenden, materiellen oder monetären Mittel, aber trotzdem gibt es bei allem keine Garantie auf irgendwelche Versprechen.
Das soziale Dienstleistungswesen birgt reichlich Besonderheiten und Widersprüche. Eine systematische Vorstellung soll helfen, den Überblick nicht zu verlieren, wenn man auf Details eingeht (vgl. Abb. 1). Soziale Leistungen haben einige wesentliche Elemente:

- An sozialen Diensten sind Interessengruppen beteiligt. Die lassen sich einer bestimmten Hierarchie zuordnen und so können ihre Rollen und Funktionen erkannt und bearbeitet werden. Auf der Mikroebene sind potenzielle und tatsächliche Leistungsempfänger zu finden, die Mesoebene ist der betriebliche Handlungsbereich, auf der Makroebene agieren das politische Gemeinwesen und Leistungsträger und das nicht unmittelbar beteiligte Umfeld bildet eine Metaebene. Vom diesem Schema ausgehend lassen sich die Eigenschaften der Akteure verfeinern und ihre Aufgaben bestimmen.

- Soziale Dienste kommen in typischen Stufen zustande. Im Sozialbereich erscheint die Erstellung der Leistungen dreigeteilt (vgl. Abb. 2). Zunächst geht es um Vorbereitung oder Anbahnung, um den Bedarf an Hilfe und den Input an Arbeitsmitteln. Es folgen die Bereitstellung der Ressourcen, Einsatz und Verfügbarkeit der Dienste. Erst mit tatsächlicher Beanspruchung werden Leistungen erbracht, Wirkung
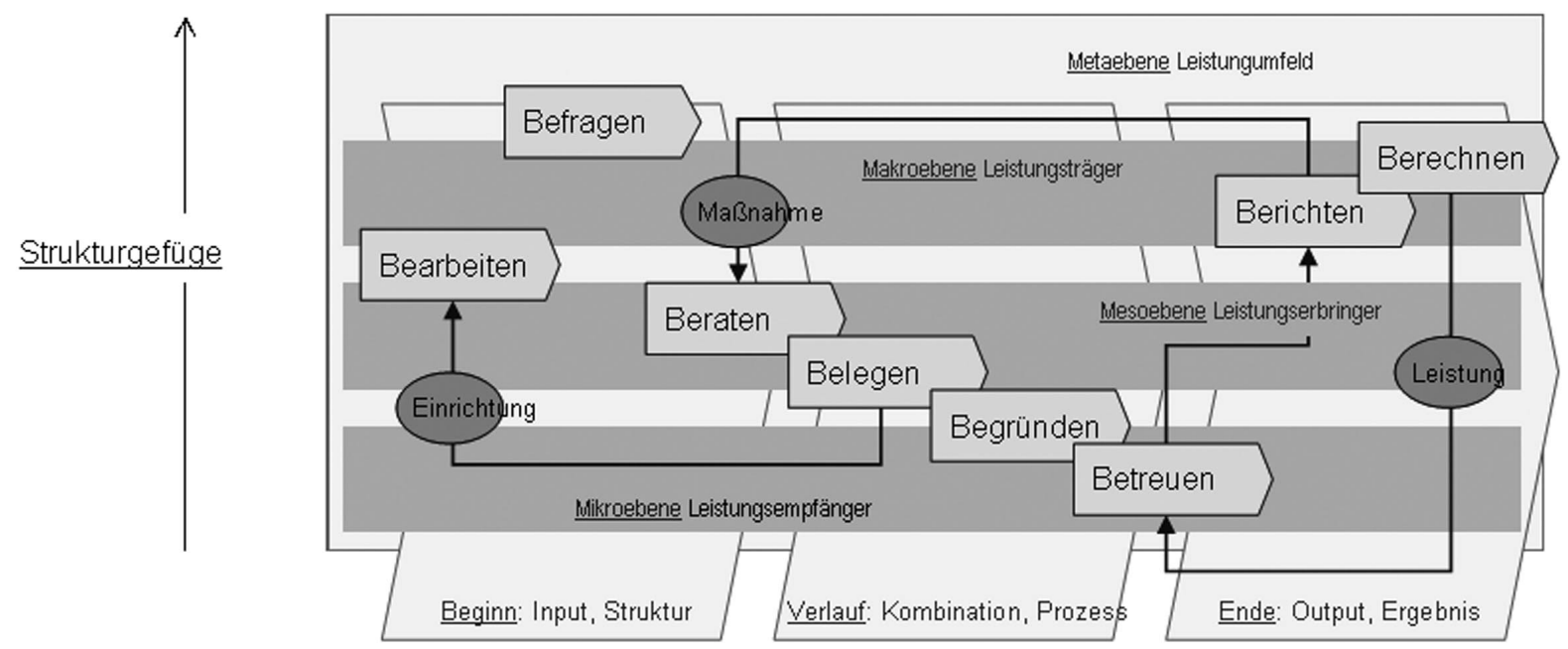

Verlaufsgeschehen

Abb. 1: Das Funktionsmodell versucht die verschiedenen Elemente sozialer Dienstleitungen in Beziehung zu setzen. 
und Ergebnis erzielt. Über diesem Verlauf liegen typische Leistungsphasen sozialer Dienste und die lassen sich bis zur Beschreibung konkreter Arbeitsschritte weiter ausdifferenzieren.
- Soziale Dienste haben spezifische Eigenschaften. Ein Sozialbetrieb oder eine ganze Branche haben viele Leistungsgegenstände und die müssen geordnet und beschrieben werden. Das gesamte Sortiment

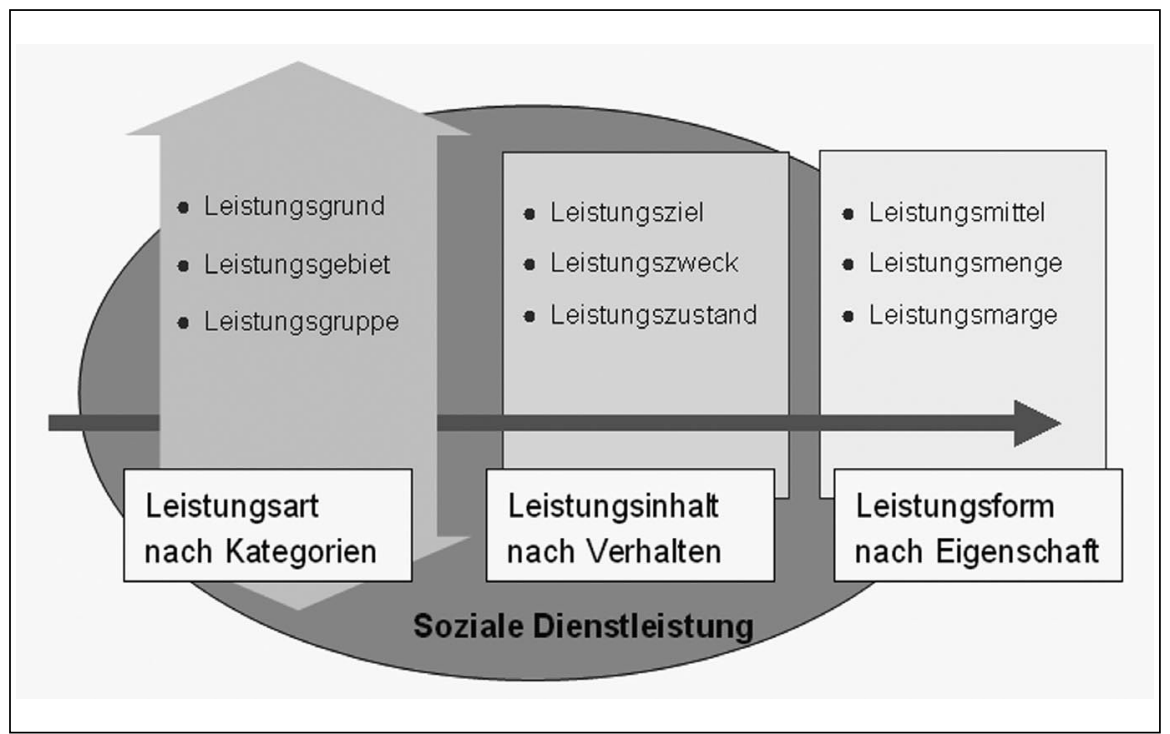

Abb. 2: Die systematische Beschreibung fasst die drei typischen Stufen einer sozialen Dienstleistung zusammen.

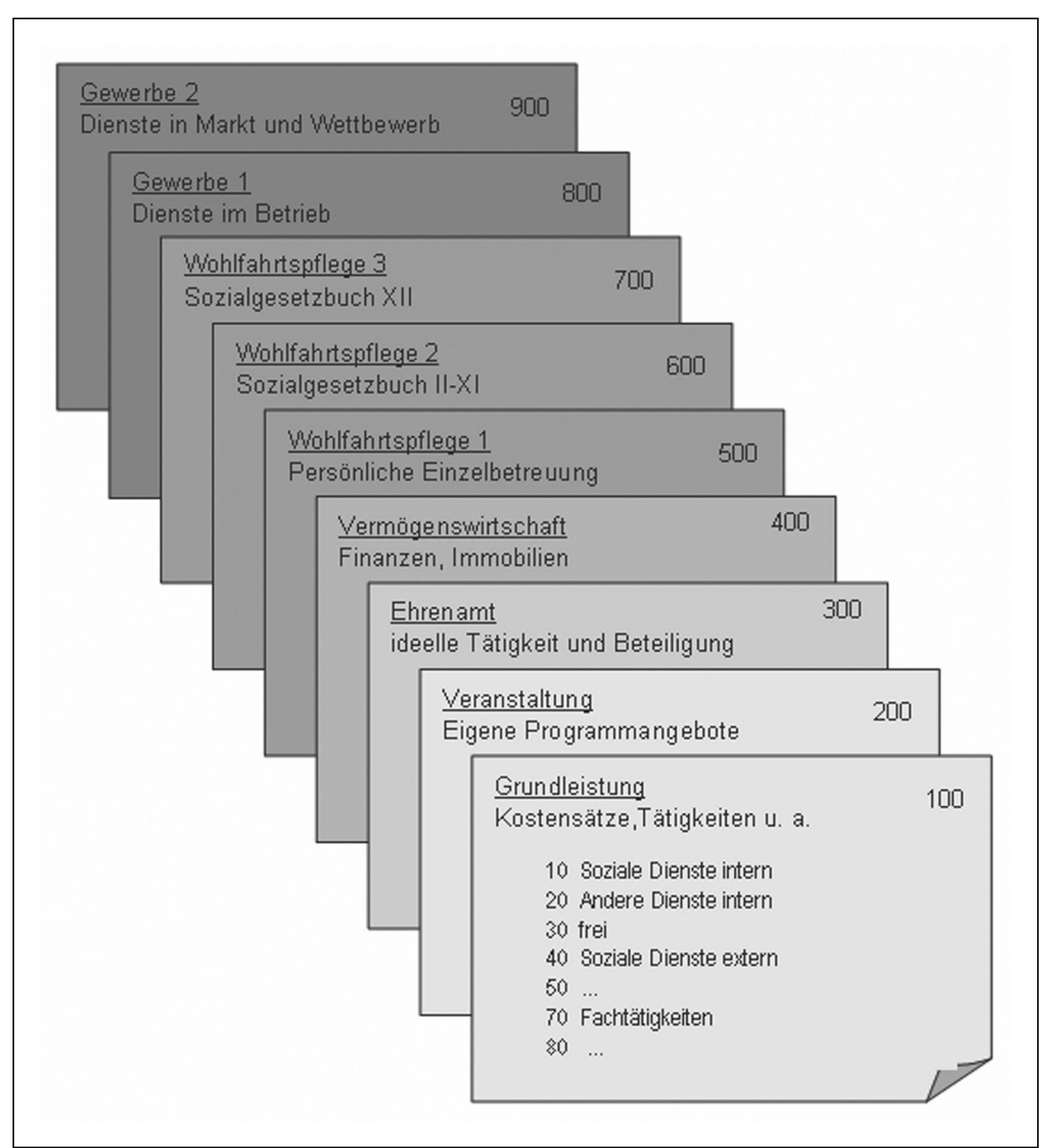

Abb. 3: Das Leistungsprogramm eines sozialen Dienstes fasst alle Angebote nach sinnvollen Leistungsgruppen zusammen. lässt sich erst einmal nach sinnvollen Leistungsarten und logischen Kategorien unterteilen. Jeder einzelne Service, jedes greifbare Produkt oder jede Geldzahlung wird anhand typischer Eigenschaften gekennzeichnet. Unterschieden nach Leistungsinhalt und Leistungsform sind alle wichtigen Parameter aufgenommen und lassen sich für verschiedene Zwecke beliebig abrufen.

In diesem Funktionsmodell ergibt sich aus den Ebenen und Stufen eine Matrix, in der sich Hilfeleistungen nach bestimmten Mustern quasi bewegen, sich mit einheitlichem Verfahren und recht exakt beschreiben lassen. Wie weit man damit geht, wie differenziert die Einzelheiten ausgewiesen werden, ist eher eine Frage des Nutzens als der Machbarkeit. Entscheidend ist zunächst, dass das Gesamtsystem stets konsistent ist. Erhebliche Mühe bereitet es, die einzelnen Elemente sauber auseinander zu halten, sinnvolle Kategorien zu finden und verständlich zu bezeichnen, Standards und logische Abgrenzungen zu bestimmen. Das alles vertieft das Verständnis der sozialen Dienste und öffnet gleichermaßen die Türe zur fachlichen Qualifizierung wie auch zur standardisierten Verarbeitung der vielen Informationen und Daten.

\section{Am praktischen Nutzen wird noch gearbeitet}

Soweit die Theorie. Aus dem Modell ist inzwischen ein regelrechtes Leistungsprogramm für die Praxis entstanden, teilweise war es wohl auch umgekehrt. Jedenfalls hat LEBENSRÄUME damit einige Impulse zur Weiterentwicklung seiner sozialen Dienste erhalten und es besteht die realistische Aussicht, bald ein funktionierendes Leistungsmanagement für soziale Dienste zu haben. Das wird nötig sein, um den heute erwarteten personenzentrierten Hilfen und der Durchmischung unterschiedlicher Lebens- und Bedarfszustände geeignete Bausteine an realen sozialen Leistungen gegenüberstellen zu können. Das wird gebraucht, um Sozialbetriebe leistungsfähig zu machen, damit sie aktuell erkannte Bedarfe befriedigen, Nachfrage decken können.

Viele Möglichkeiten einer effektiven und effizienten Steuerung erge- 
ben sich, wenn man den modularen Umgang mit sozialen Diensten beherrscht. Auf die Anfrage eines Budgetnehmers kann man eben schlecht mit einem Wohnheim antworten und manchmal nutzt ein diskretes Päckchen aus Sicherheitsversprechen, Gemeinschaftseinbindung und sinnvoller Beschäftigung mehr als massiv therapeutisch rehabilitative Intervention. Angesichts der Zunahme psychischer Erkrankungen im Arbeitsleben könnten modifizierte Betreuungsformen der Behindertenhilfe auch hier dienlich sein usw.

Beispiele zeigen, wie das Management der Hilfeleistung in den Vordergrund der sozialbetrieblichen Steuerung tritt und das sind Teile davon:

- Im Leistungsprogramm sind alle Dienste hinterlegt. Ein umfangreicher Katalog weist beliebig viele Leistungen aus, vom einzelnen Baustein über Maßnahmebündel bis zu komplexen Einrichtungen (vgl. Abb. 3). Das Ordnungssystem folgt der Leistungsart (Grund, Gebiet, Gruppe) und relevante Merkmale sind nach Leistungsinhalt (Ziel, Zweck, Zustand) und Leistungsform (Mittel, Menge, Marge) hinterlegt. Eine Liste verschafft Übersicht, jede einzelne Leistung lässt sich mit einem Datenblatt exakt darlegen, Verfahren zur Personalbemessung und Kostenkalkulation ergänzen das Tabellenwerk.

- In der Kundenakte werden Leistungsdaten dokumentiert. Im Verlaufe der sozialen Dienste ist eine typische Folge von Arbeitsschritten zu erbringen und immer wieder sind gleiche Leistungsdaten aufzurufen: Zeitangaben machen, Vergütung berechnen, Bericht geben etc. Auf Basis von Modell und Leistungsprogramm gibt es eine einheitliche Kundenakte, in die operative Angaben standardisiert und formalisiert aufgenommen werden. Strukturierte Vorgaben leiten das Personal fachlich administrativ an, Informationen sind auf die Person bezogen dokumentiert und schon zur Weiterverarbeitung geordnet.

- Integrierte Software soll die Leistungsdaten verarbeiten. Die Digitalisierung sozialer Dienste führt zu einer Menge verknüpfter Daten. Mithilfe moderner Informationstechnologie sollte sich das
Leistungsprogramm durch geeignete Software verwalten lassen, das gelingt noch nicht. Fachbezogene Programme erweisen sich als wenig universell, betriebswirtschaftliche Standardmodule verarbeiten nur Einzelfunktionen. Auch eine Eigenprogrammierung scheint wenig aussichtsreich. Nun wird versucht, eine integrierte, internetbasierte Mittelstandssoftware von SAP für soziale Dienste zu erschließen.

Interessant ist die Umkehr des Steuerungsansatzes, der aus gutem Grund erfolgt. Der Personaleinsatz per Stellenplan war lange Ausgangspunkt fast jedes sozialen Dienstes und die mögliche Leistung ergab sich dann, je nach dem, was daraus gemacht wurde. Für bestimmte Personengruppen und Lebenslagen wurden Institutionen wie Heimeinrichtungen, Werkstätten, Beratungsstellen konzipiert und die Vielfalt sozialer Hilfestellungen ließ sich, derart reduziert, ganz gut betrieblich beherrschen.

Nun verändern sich die Prämissen. Individuelle Lebenssituationen stehen mit Zielen der Integration, Teilhabe und Selbstbestimmung im Vordergrund und die Steuerung der reinen Facharbeit erhält einen ganz neuen Stellenwert. Nicht alles darf man sich da als machbar vorstellen, aber gerade die fachliche Seite der Sozialen Arbeit sollte zukünftig noch erheblich vom sozialwirtschaftlichen Sachverstand profitieren können. Das ist freilich noch immer ungewohnt und reichlich mühsam.

\section{Ihr Kontakt zur SOZIALwirtschaft ...}

... für Leserinnen und Leser, die Artikel kommentieren wollen:

Gerhard Pfannendörfer, Chefredaktion

SOZIALwirtschaft

E-Mail pfannendoerfer @nomos.de

... für Autorinnen und Autoren, die ein Thema vorschlagen wollen:

Gerhard Pfannendörfer, Chefredaktion

SOZIALwirtschaft

E-Mail pfannendoerfer @nomos.de

... für Leserinnen und Leser, die ein Abonnement bestellen wollen:

Aloisia Hohmann, Nomos Verlagsgesellschaft E-Mail hohmann@nomos.de

... für Leserinnen und Leser, die ein Einzelheft bestellen wollen:

Aloisia Hohmann, Nomos Verlagsgesellschaft E-Mail hohmann@nomos.de

... für Organisationen und Agenturen, die eine Anzeige oder eine Beilage schalten wollen:

Kerstin Ternes, Agentur sales friendly E-Mail ternes@salesfriendly.de

... für Leserinnen und Leser, die ein kostenloses Ansichtsexemplar des Informationsdienstes SOZIALwirtschaft aktuell bestellen wollen:

Aloisia Hohmann, Nomos Verlagsgesellschaft E-Mail hohmann@nomos.de

... für Leserinnen und Leser, die sich über das Buchprogramm von Nomos informieren wollen: Internet http://nomos.de 